\title{
Cancellation of Matched Filter Sidelobes for LFM Radar using Regularized Orthogonal Matching Pursuit
}

\author{
Sudha Hanumanthu, P.Rajesh Kumar
}

\begin{abstract}
Major disadvantage of matched filter is with sidelobes as they might cause false alarms or may mask weak targets of radar echo. In applications like Urban sensing, Through the wall radar, Non destructive testing etc small objects of interest play crucial role which might be masked by sidelobes of matched filter or sometimes sidelobes may be mistreated as objects of interest that lead to false alarms. Many solutions have been proposed in the literature to suppress these sidelobe levels to some extent but not completely cancelled. In this paper, a new approach is utilized to completely cancel the sidelobes of matched filter using a greedy algorithm known as Regularized Orthogonal Matching Pursuit (ROMP). This algorithm is applied to the matched filter output that resulted in complete removal of sidelobes. Also SNR is improved. Even at very low SNRs targets are detected with high probability and hence the proposed approach is more robust to noise.
\end{abstract}

Keywords : Matched Filter, Sidelobes, ROMP, SNR

\section{INTRODUCTION}

In pulse radar, range resolution is inversely proportional to bandwidth. If width of the pulse is reduced bandwidth is increased but average transmission power decreases which limit the maximum unambiguous range of targets and also resolution. In order to improve range resolution without reducing pulse width, one most familiar signal processing technique used is correlation processing also called as Matched Filtering. Here the transmitted pulse is modulated using either frequency or phase modulation that results in a narrow pulse at the output of the Matched Filter (MF) with a high peak value. Major disadvantage with $\mathrm{MF}$ is that correlation function between transmitted and received signals produces very high sidelobes [1]. These may mask weak targets or may be mistreated as targets (false alarms).

A reduction of sidelobes can be accomplished by applying a window function. But this leads to loss in peak value i.e. SNR decreases and also main lobe width increases which leads to loss in resolution [2]. Also as in [3] mismatched filters can be used where standard convex optimization algorithms are applied and Integrated Sidelobe Level is

Revised Manuscript Received on April 21, 2020.

* Correspondence Author

Sudha Hanumanthu*, Department of ECE , Andhra University College of Engineering, Visakhapatnam, India. Email: sudha.klce@gmail.com

P. Rajesh Kumar, Department of ECE , Andhra University College of Engineering, Visakhapatnam, India. Email: rajeshauce@gmail.com

(C) The Authors. Published by Blue Eyes Intelligence Engineering and Sciences Publication (BEIESP). This is an open access article under the CC BY-NC-ND license (http://creativecommons.org/licenses/by-nc-nd/4.0/) reduced by minimizing the 11-norm of the vector whose elements are the sidelobe energies. This approach is more computationally complex than the Matched Filter because filter weights need to be estimated for each range bin separately.

In [4],[5] target detection and estimation parameters are used to compare different apodization techniques applied on LFM matched filter output with the NLFM matched filter output. As observed so far all the past research could reduce sidelobe level to some extent while compromising for loss in resolution and SNR.

In this paper a new approach Orthogonal Matching Pursuit (ROMP) [6][7], which is a fast iterative greedy algorithm is used that cancels the sidelobes completely without compromising for loss in resolution.

The content organization of the paper is as follows. First in section II, a brief theory of MF frame work is provided and a detailed outlook of the methodology used in this paper is given. In section III ROMP algorithm for sidelobe cancellation of targets of MF output is proposed. In section IV simulation results are presented and in section $\mathrm{V}$ concluding remarks are made.

\section{PULSE COMPRESSION}

Using long pulses we can transmit the pulse to cover targets that are far away from transmitter. But as long pulse has limitation of low resolution close targets can't be separated at the receiver output. This drawback can be overcome using pulse compression technique.

In a pulse compression radar long pulse will be transmitted via frequency or phase modulation or digitally via Costas, Barker or Frank codes [8] that results in a short pulse at the output of the Matched Filter receiver achieving high range resolution. The modulation used in transmitted signals can be frequency or phase modulation.

Fig.1. below shows classification of different modulation schemes used in transmitted waveforms for pulse compression.

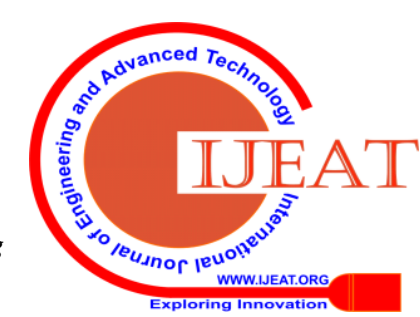




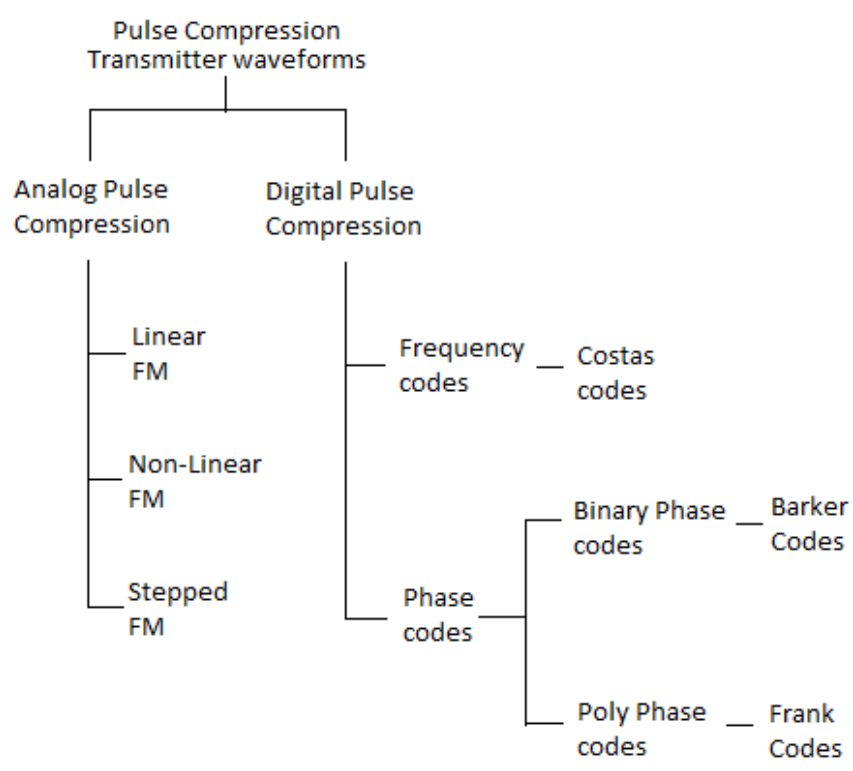

Fig.1. Pulse compression waveforms

\section{A. Theory of Matched Filter}

Closely spaced targets cannot be distinguished or resolved by processing unmodulated signals through matched filter i.e. resolution will be very poor. For pulse compression to occur, phase or frequency modulated signals should be processed through matched filters. The echo signal when passed through filter matched to the transmitted signal produces a much narrower, sharp peak in the output. Resolution as well as SNR is improved by a matched filter.

Processing a signal through matched filter is equivalent to computing cross-correlation between transmitted modulated signal and received echo signal from target. Correlation is performed digitally via Fast Fourier Transform also known as Fast convolution processing. The impulse response of the digital matched filter for a specified input signal, $\mathrm{x}(\mathrm{t})$, is given by Eq. (1)

$$
\mathrm{h}(\mathrm{k})=\operatorname{IFFT}\left\{[\operatorname{FFT}(\mathrm{x}(\mathrm{k}))]^{*}\right\}
$$

Where $\mathrm{x}(\mathrm{k})$ is the sampled version of $\mathrm{x}(\mathrm{t})$ and $\mathrm{h}(\mathrm{k})$ represents filter coefficients. FFT and IFFT mean Fast Fourier Transform and Inverse Fast Fourier Transform respectively. Fig.2. below shows block diagram of Matched filtering operation.

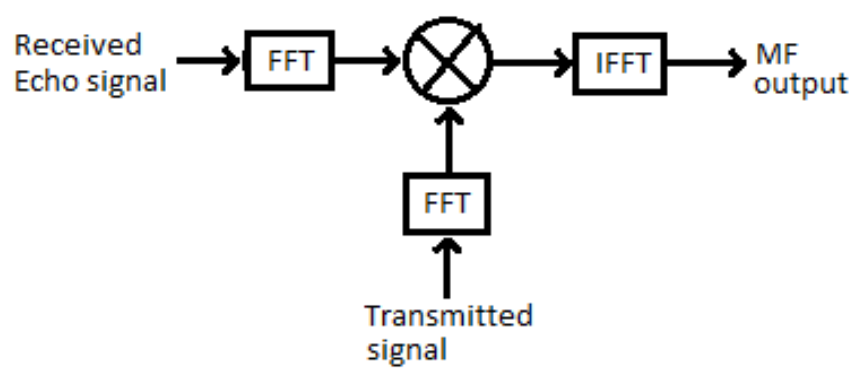

Fig.2. Matched Filtering

Linear Frequency Modulated signal

LFM signal is commonly used to achieve wider bandwidth since a long pulse can have same bandwidth as a short pulse if modulated in frequency or phase. A typical LFM waveform can be expressed by Eq. (2)

$$
r(t)=\operatorname{Rect}\left(\frac{t}{\tau_{0}}\right) e^{j\left(2 \pi \pi_{0} t+C \pi t^{2}\right)}
$$

Where the pulse width is $\tau_{0}, C=B / \tau_{0}, \mathrm{~B}$ is the bandwidth, $f_{o}$ is the radar center frequency. $\operatorname{Rect}\left(t / \tau_{0}\right)$ is rectangular pulse of width $\tau_{0}$.

\section{Proposed Approach}

\section{A. Regularized Orthogonal Matching Pursuit (ROMP)}

A greedy algorithm known as ROMP is applied to the output of matched filter to cancel the sidelobes completely. Resolution is not affected. ROMP is generally used to solve under determined systems to get sparse solution. Consider the Eq. (3) with $\mathrm{z}$ representing incomplete measurements of MF output $y$,

$$
z=\phi \times y
$$

Where $\mathrm{y}$ is matched filter output of order $\mathrm{n} \times 1, \Phi$ is Fourier matrix of order $m \times n$ and $m<<n$.

In Fig.3, below block diagram of the proposed method is given.

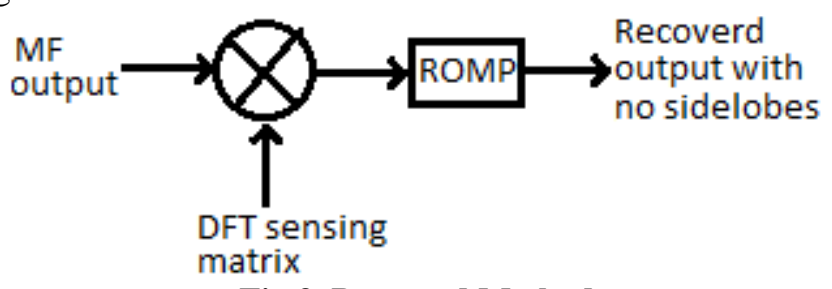

Fig.3. Proposed Method

\section{B. Probability of detection}

Signal processing system has two tasks: $1^{\text {st }}$ task is to detect the presence of signal in noise. $2^{\text {nd }}$ task is information extraction [9]. Detection theory provides the foundation for $1^{\text {st }}$ task i.e. it says whether target (targets in multiple target scenario) is present or only noise is present in the received signal. Estimation theory deals with $2^{\text {nd }}$ task i.e. it gives information about location (range) of targets, Doppler shift etc. One cannot move to $2^{\text {nd }}$ task unless $1^{\text {st }}$ task is fulfilled i.e. without targets present no meaning in estimation of targets.

Detection theory implements hypothesis testing.

H0: Receives signal=Noise alone

H1: Receives signal $=$ Target + Noise

Detection after MF is based on whether the power or magnitude of MF output is greater or lesser than threshold $v_{t h}$, given by Eq. (4)

$$
v_{t h}=\sqrt{-2 \sigma^{2} \ln p f a}
$$

Where $\sigma^{2}$ is the variance of noise and $p f a$ is the probability of false alarm which represents probability that received signal exceeds the threshold under hypothesis H0. Probability of detection (Pd) is the probability that received signal exceeds the threshold under hypothesis H1 [10].

\section{SIMULATION RESULTS}

In this section experimental simulations are presented and analysis is made. The transmitted signal used is an LFM waveform with center frequency $\mathrm{f}_{0}$ set to zero, pulse width tau of $1 \mu$ s and a bandwidth, $b$ of $30 \mathrm{MHz}$. Transmitted LFM chirp pulse is shown below in Fig.4.

Published By:

Blue Eyes Intelligence Engineering \& Sciences Publication 


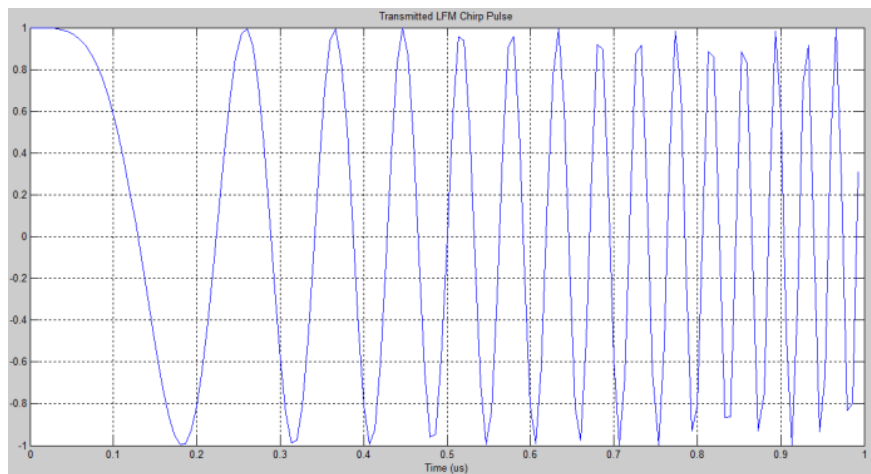

Fig.4. LFM chirp pulse

Four target positions are set at $100 \mathrm{~m}, 203 \mathrm{~m}, 380 \mathrm{~m}$ and $620 \mathrm{~m}$ with amplitudes $1,0.7,0.6$ and 0.8 respectively. Composite received echo signal is shown below in Fig.5.

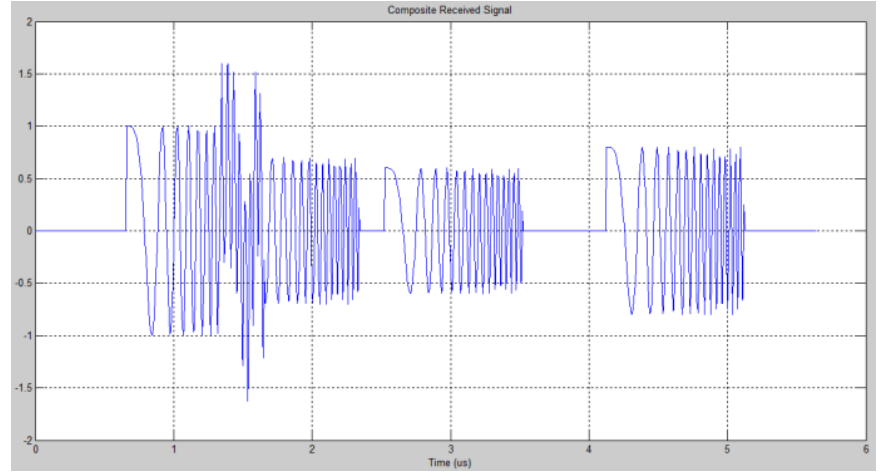

Fig.5. Composite received echo signal

Matched Filter output and recovered output after applying ROMP algorithm to matched filter output is shown below in Fig.6. As can be seen from the result that sidelobes are totally eliminated using the proposed approach.

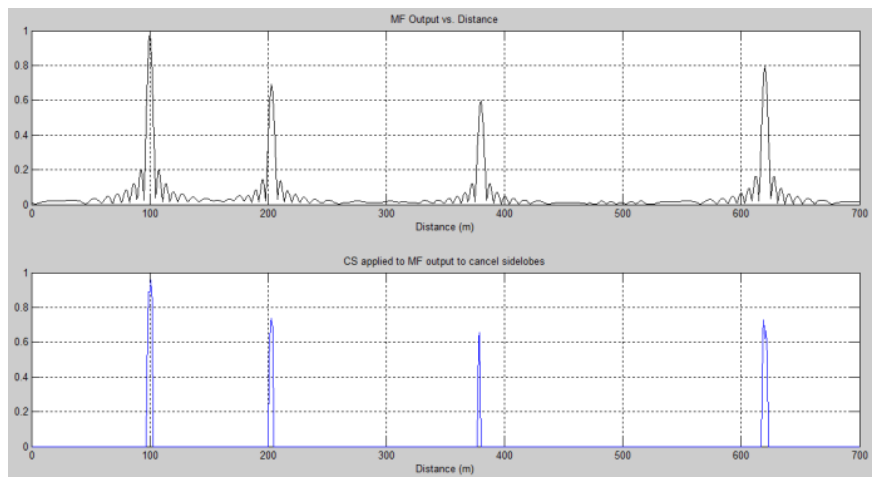

Fig.6. Sidelobes cancellation

Sample ratio is given by, $\mathrm{SR}=\mathrm{n} / \mathrm{m}$, where $\mathrm{n}$ is number samples of composite received signal and $\mathrm{m}$ is the number of rows in DFT sensing matrix. For different values of $\mathrm{m}$, using Marcum function with probability of false alarm, $\mathrm{pfa}=0.001$, detection probability is measured and plotted against SR as shown below in Fig.7.

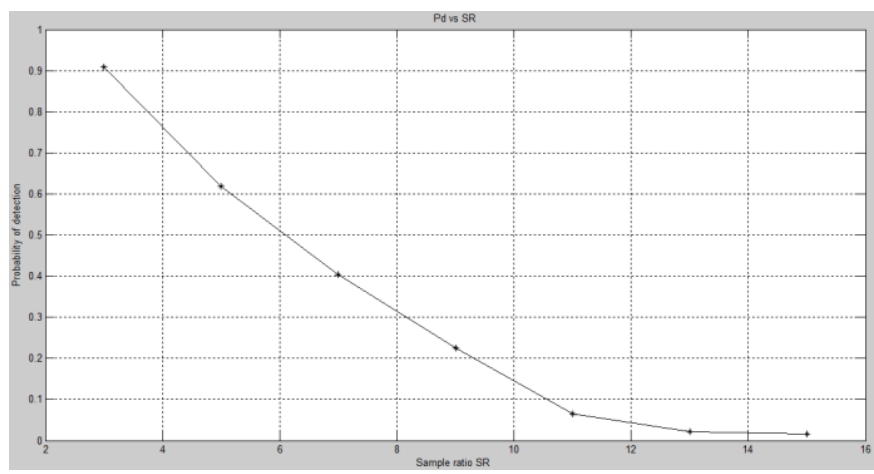

Fig.7. Probability of detection vs. Sample ratio

It can be noticed that as $\mathrm{m}$ value decreases or $\mathrm{SR}$ value increases, probability of detection of targets decreases . For $100 \%$ detection of targets value of $m$ to be taken is 100 and above or SR value is below 8 .

To observe the detection probability of the proposed method in presence of noise, simulations are performed by adding noise to the received echo signal.

In the Fig. 8 below SNR=-10dB added to the echo signal is shown.

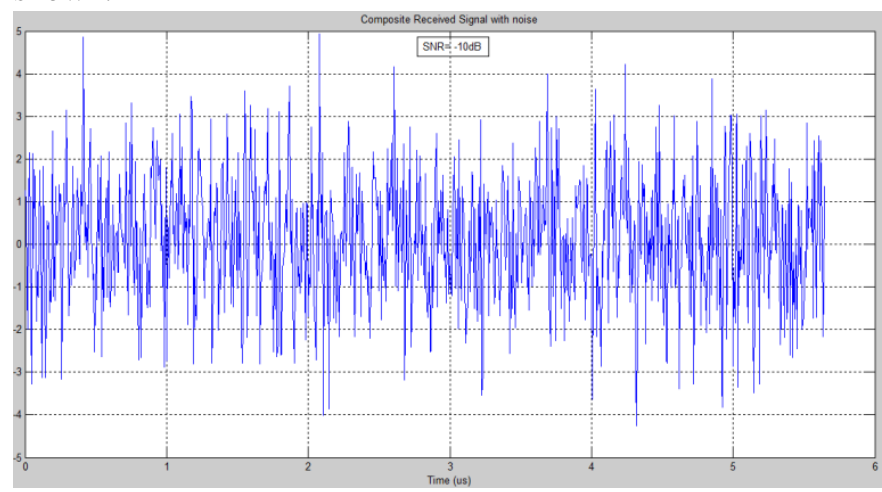

Fig.8. Composite received echo signal with noise

The MF output as well as output of ROMP algorithm after applied to MF output at $\mathrm{SNR}=-10 \mathrm{~dB}$ noise is shown in Fig.9.

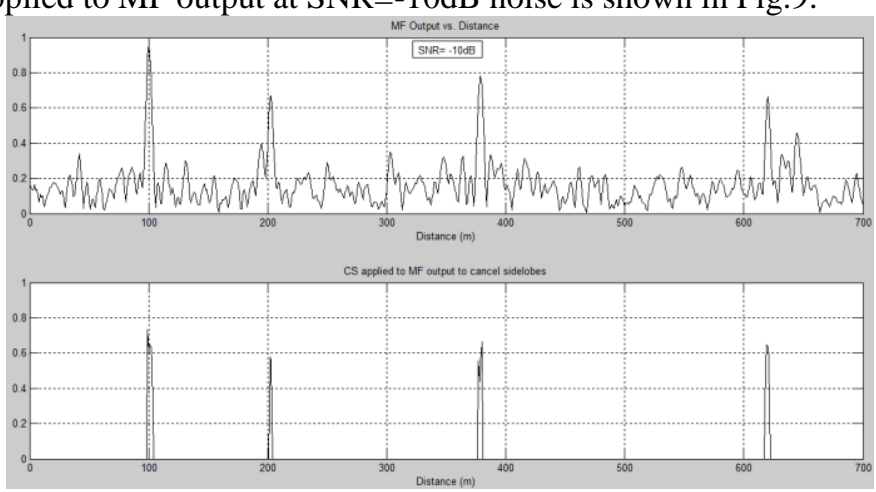

Fig.9. Sidelobes cancellation in presence of noise

As can be seen from the above result that the proposed approach is very effective in estimation of target locations even in the presence of high noise i.e. at $\mathrm{SNR}=-10 \mathrm{~dB}$. Noise along with sidelobes has been totally eliminated.

Detection performance in presence of noise for different values of SNR is plotted as shown below in Fig.10.

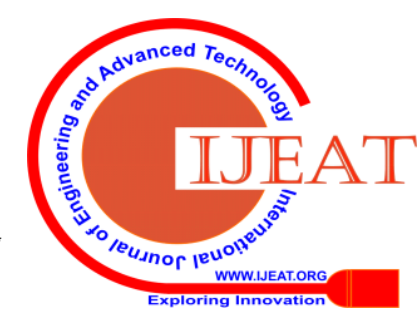




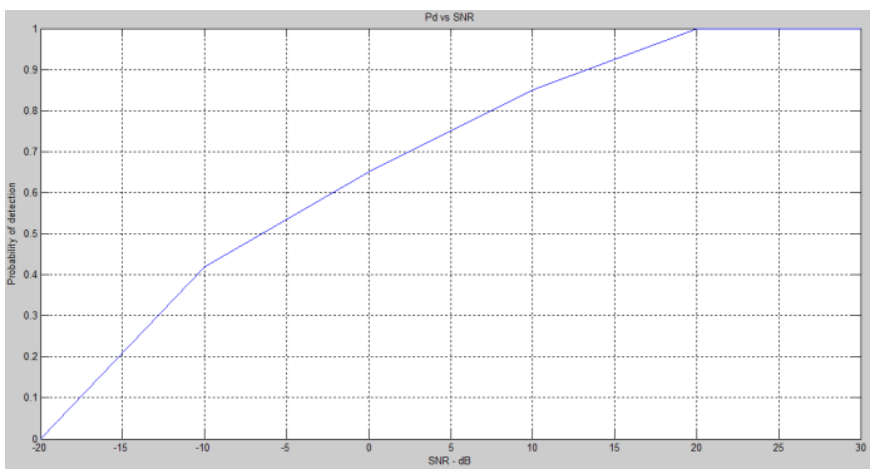

Fig.10. Probability of detection vs. Signal to Noise ratio

\section{CONCLUSION}

The main advantage of MF is its ability to improve SNR even in presence of high noise. But the disadvantage of $\mathrm{MF}$ sidelobes which may mask weak targets or appear to be false alarms can be overcome using the proposed approach. ROMP algorithm applied to the MF output is effective in removing sidelobes completely. Resolution is same as that of MF while SNR is improved beyond MF. Targets can be detected as well as estimated with high probability even at very low SNRs using the proposed approach.

\section{REFERENCES}

1. F.Kreetschmer Jr. and K. Gerlach, "Low sidelobe radar waveforms derived from orthogonal matrices, "IEEE Transactions on Aerospace and Electronic Systems, vol.27, no.1, pp.92-102, Jan 1991.

2. Shlan R. Dffiraaf, "Sidelobe reduction via adaptive FIR filtering in SAR imagery," IEEE Tram. Image Processing, vol. 3, pp. 292-301,May 1994.

3. Ackroyd M.H. and F. Ghani. "Optimum mismatched filters for sidelobe suppression," IEEE Transactions on Aerospace and Electronic Systems. AES-9 (Mar. 1973), 214-218.

4. C. Vizitiu, "Sidelobes reduction using synthesis of some NLFM laws," Progress In Electromagnetics Research B, Vol. 49, pp. 301-318.

5. L. R. Varshney and D. Thomas. Sidelobe reduction for matched filter range processing. In Proc. IEEE Radar Conf., 2003.

6. Joel A Tropp and Anna C Gilbert, "Signal recovery from random measurements via orthogonal matching pursuit," IEEE Transactions on Information Theory, vol. 53, no. 12, pp. 4655-4666, 2007.

7. D. Needell and R. Vershynin. "Signal recovery from incomplete and inaccurate measurements via regularized orthogonal matching pursuit," IEEE Journal of Selected Topics in Signal Processing, 4(2):310 -316,April 2010.

8. Rajasekhar Manda, Rajesh Kumar P, "Polyphase sequence analysis with good merit factor and correlation properties", Journal of Engineering Science and Technology, Vol. 13, No. 12 (2018) 4160 4172.

9. Steven M.Kay, "Fundamentals of statistical signal processing, volume 2, Detection Theory" Prentice Hall signal processing series.

10. Bassem R. Mahafza, "Radar Systems Analysis And Design Using Matlab" 3e, CRC Press, Taylor \& Francis Group, ISBN-13: 978-1-4398-8496-6.

\section{AUTHORS PROFILE}

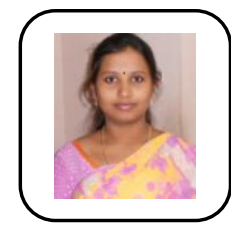

processing.

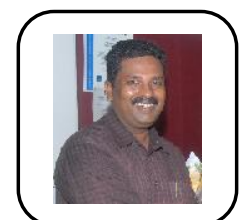

P. Rajesh Kumar received B.E degree from Osmania University, Hyderabad. M.E degree from Andhra University, Visakhapatnam. He has teaching experience of more than 20 years in various Engineering colleges. He is currently working as

Visakhapatnam. Her research area is Radar signal
Published By:

Blue Eyes Intelligence Engineering \& Sciences Publication

(C) Copyright: All rights reserved. 